\title{
Visualization of Large-scale Atomic Interactions During the Melting and Crystallization Process
}

\author{
ROMAN DัURIKOVIČ* \\ Department of Computer Software, The University of Aizu, Aizu-Wakamatsu, Fukushima-ken 965-8580, Japan
}

\begin{abstract}
Atomic-scale material model capable of melting, crystallization and amorphization has been developed to examine the defect formation and crystal growth processes from melted silicon $(\mathrm{Si})$ based on the ordinary Langevin equations of motion. The developed computer system consists of simulation and visualization part. Simulation supports the large-scale molecular-dynamics (MD) clusters with solid/liquid interface responding interactively to the control parameters such as the temperature gradient and pulling speed. Material behaviour simulation is limited to $10^{4}$ particle objects representing different atoms. A particle in proposed dynamic system interacts through attractive covalent forces and short-range repulsion forces in all three dimensions. This research was conducted to understand the processes that can control the quality of single-crystal Si grown from the melt by Czochralski crystal puller.
\end{abstract}

Keywords: Molecular dynamics; Si crystallization; Czochralski puller

\section{INTRODUCTION}

Although, defect formation can be hardly avoided in $\mathrm{Si}$ technologies such as crystal growth from melted $\mathrm{Si}$, and thermal oxide growth, their topology as well as formation mechanism are not well understood, because of high temperature and intensive reactivity of melt. Molecular dynamics (MD) is currently the only available method to handle the large-scale simulations of solidification from melted Si. Small molecular models (up to 100 atoms) that may contain multiple types of atoms, can be created with a commercial quantum mechanics package called Gausian94 [1]. This package falls in to a group of ab initio methods when very accurate molecular orbital calculations are carried out for the entire structure with hydrogen termination. Unfortunately, no such system is available for larger molecular models.

Over the years, much attention has been given in the physics and chemistry literature to the development of discrete liquid models involving aggregate molecular dynamics in which the molecules are subject to various interaction potentials, viz. Berendsen [2]. A basic technique is to model longrange attraction and short-range repulsion forces between pairs of particles according to potentials of the Lennard-Jones type, which lead to forces

*Tel.: (242) 456-7890, Fax: (321) 654-0987, e-mail: roman@u-aizu.ac.jp 
involving inverse powers of particle separation distance as proposed by Tersoff [3]. Recently Ishimaru et al. [4] demonstrated that interatomic potential proposed by Tersoff is very good in reproduction of experimental results and in addition to crystal $\mathrm{Si}$ simulations it can be used to simulate both the amorphous and liquid state of pure Si by different heating and freezing methods of melted Si. This paper proposes the approach for modeling and visualization of large scale molecular models applied on the simulation of a singlecrystal silicon growth from molten silicon. To provide experimentalists with a familiar way of looking at structure of simulation data, several diagnostic imaging techniques that aid the analysis of structure at atomic level such as radial distribution function, bond angle distribution function, and static structure factors are proposed here to be calculated from particle clusters in a run.

\section{SIMULATION METHOD}

In order to simulate the pulling motion of a crystal the Langevin equation of motion should be slightly modified by introduction of a constant pulling speed $\mathbf{v}^{c}$, that is

$$
\begin{aligned}
& m_{i} \dot{\mathbf{v}}_{i}(t)=-m_{i} \gamma_{i} \mathbf{v}_{i}(t)+F_{i}(\{\mathbf{x}(t)\})+R_{i}(t) \\
& \dot{\mathbf{x}}_{i}+\mathbf{v}^{c}=\mathbf{v}_{i},
\end{aligned}
$$

where $\mathbf{x}_{i}, \mathbf{v}_{i}$, and $\gamma_{i}=5 \mathrm{ps}^{-1}$, are the position, velocity, atomic mass and friction coefficient of the $i$-th particle at time $t$, respectively. The interatomic force $F_{i}$ between the $N$ particles is derived as variational derivative of the Tersoff [2] potential for $\mathrm{Si}$, the stochastic force $R_{i}$ was introduced to mimic the motion of solvent molecules on the solute, it also serves as temperature controller in the system. The use of Tersoff parameters for the $\mathrm{Si}$ and $\mathrm{C}$ type of molecules has some limitations in MD simulations as delineated by Halicionglu [5], however, they were taken into account in our implementations.
Concerning the nature of the stochastic force $R_{i}$ it is assumed to be stationary, Markovian and Gaussian with zero mean and to have no correlation with initial velocities $\mathbf{v}_{i}(0)$ nor with the systematic force $\boldsymbol{F}_{i}(0)$. The stochastic numerical integration of the third order proposed by Gunsteren and Berendsen [6] was performed under the constant volume within the cubic domain with periodic boundary conditions on side faces and a reflection plane on set at $2 \AA$ bellow the bottom of the volume. At each time step $(0.002 \mathrm{ps})$ the collision between the particle and the reflection plane is calculated to prevent particle from escaping out of the simulation volume.

\section{CRYSTAL GROWTH EXAMPLE}

For MD simulation of crystal growth, a liquidcrystal interface was at first prepared by attaching crystal sub-volume above the one with liquid atomic structure. Consequently, the system was heated at given temperature gradient along $Z$ axis and crystal atoms were pulled with constant speed in $Z$ direction for the simulation of crystal growth, see Figure 1. Figure 2 shows the simulated crystal growth process with a pulling speed of $5 \mathrm{~m} / \mathrm{s}$ in the [111] direction. Joining two heated crystalline $\mathrm{Si}$ cells made initial states. The top cell had density of crystal $\mathrm{Si}, 2.33 \mathrm{~g} / \mathrm{cm}^{3}$ and the bottom had 2.57 $\mathrm{g} / \mathrm{cm}^{3}$, which gives the density of liquid Si structure. Calculated growth rate and pulling speed are much larger then in a real crystal puller because much larger volume and longer time period is needed to
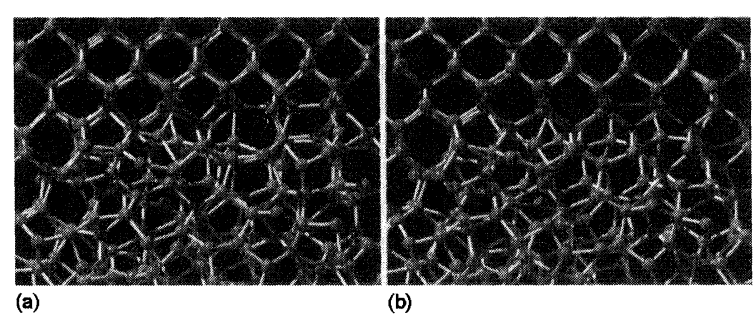

FIGURE 1 Simulation domain under consideration showing an initial liquid-crystal interface and temperature distribution along the $Z$ axis. 


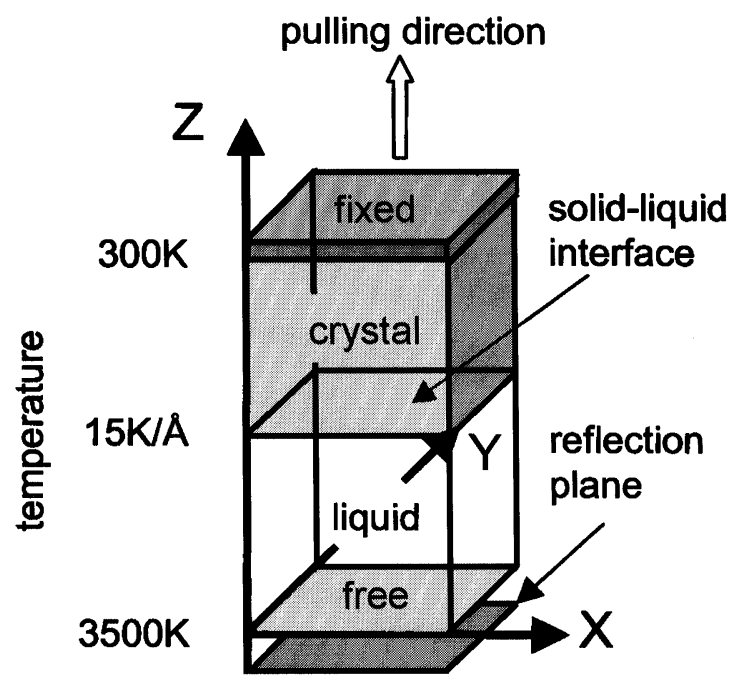

FIGURE 2 An example of atomic arrangements in crystallization processes after simulation time of a) $300 \mathrm{ps}$ and b) $600 \mathrm{ps}$.

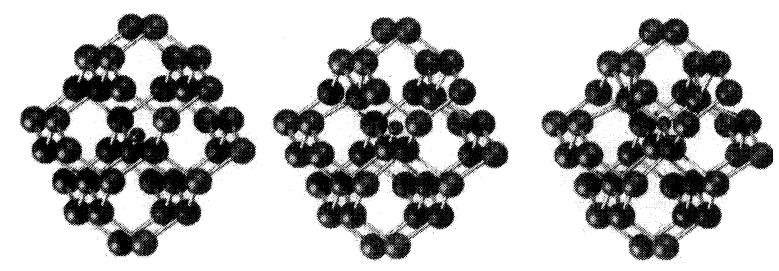

FIGURE 3 Oxygen and silicon interactions including two electron vacancies $\left(\mathrm{Si}_{36} \mathrm{O}\right)$. The oxygen atom is located in the center.

get exact results. Nevertheless, the proposed simulation system of crystallization process is useful to analyze crystal growth rates. To further study the effects of impurities on a crystal growth and defects formation we show the simulation of $\mathrm{Si}$ cluster interaction with oxygen atoms on Figure 3.

\section{CONCLUSIONS}

We have demonstrated the modeling technique of the crystallization process of liquid silicon for large-scale systems. The advantages of the proposed dynamic model are in its application for analysis and study of formation complexes of silicon defects where $a b$ initio computations may be prohibitively expensive. Implementation of additional types of atoms and their interactions in large-scale systems is under the current investigation. To get practical results with better accuracy it is important to use longer volumes and larger times.

\section{Acknowledgments}

The authors would like to thank K. Nishihira, M. Ishimaru and T. Motooka from the Department of Materials Science and Engineering, Kyushu University for their collaboration during the course of this work. This research was sponsored by grants from the Fukushima Prefectural Foundation for the Advancement of Science and Education under the project of "Scientific Visualization".

\section{References}

[1] Berendsen, H. J. C. and van Gunsteren, W. F., "Molecular dynamics simulations: techniques and approaches", In: Barnes, A. J., Orville-Thomas, W. J. and Yarwood J. (Eds.), "Molecular liquids dynamics and interactions", D. Reidel, Dordrecht, Holland, 1984.

[2] Tersoff, J. (1989). "Modeling solid-state chemistry: Interatomic potentials for multicomponent systems", Physical Review $B, \mathbf{2 3}(8)$.

[3] Ishimaru, M., Munetoh, S. and Motooka, T. (1997). "Generation of amorphous silicon structures by rapid quenching: A molecular-dynamics study", Physical Review $B, \mathbf{5 6}(23)$.

[4] "Gaussian 94 Revision E.2", Gaussian, Inc., Pittsburgh, PA, 1995.

[5] Halicioglu, T. (1995). "Comparative study on energy and structure-related properties for the (100) surface of $\beta$-SiC", Physical Review B, 51(11).

[6] van Gunsteren, W. F. and Berendsen, H. J. C. (1982). "Algorithms for brownian dynamics", Molecular Physics, 45(3). 

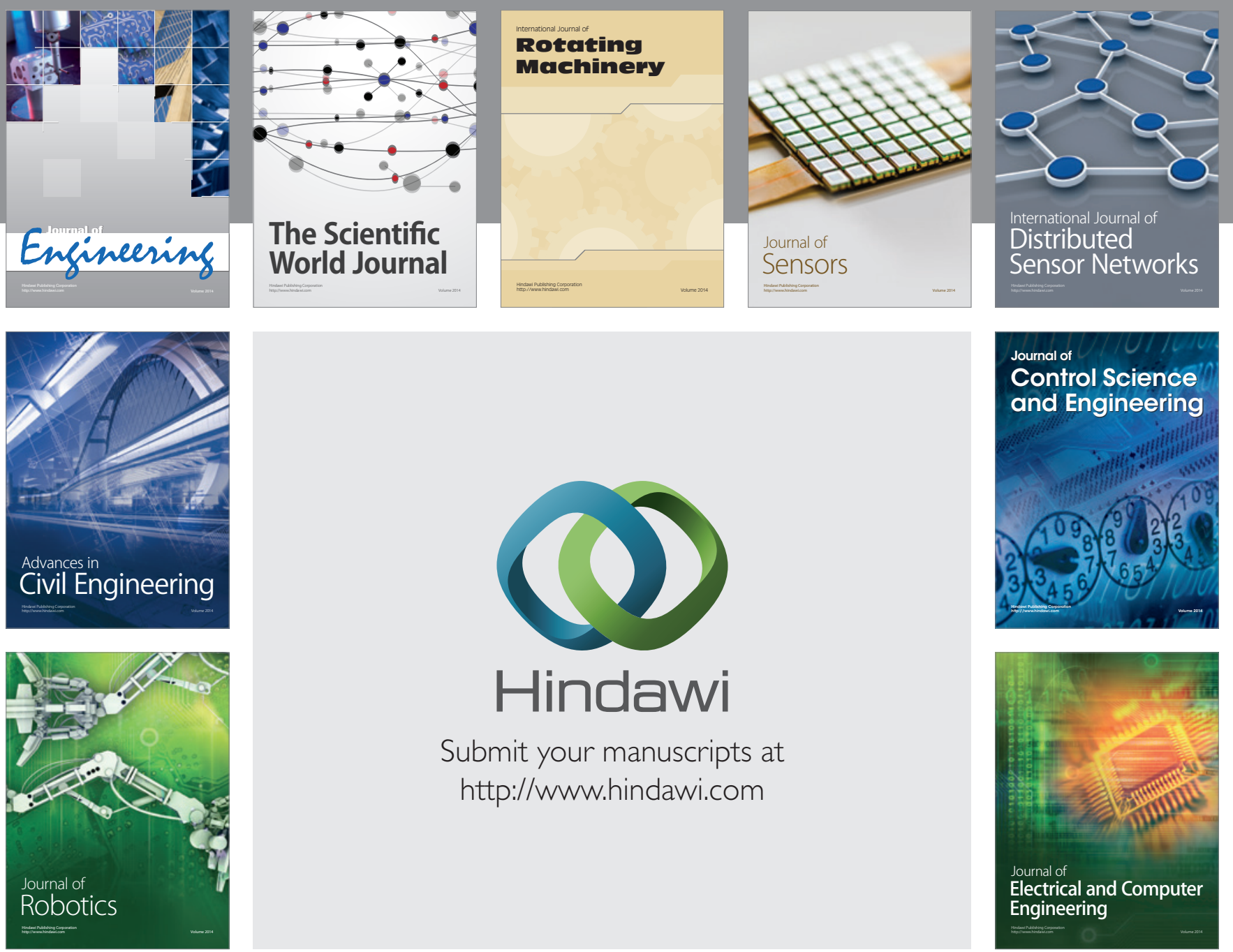

Submit your manuscripts at

http://www.hindawi.com
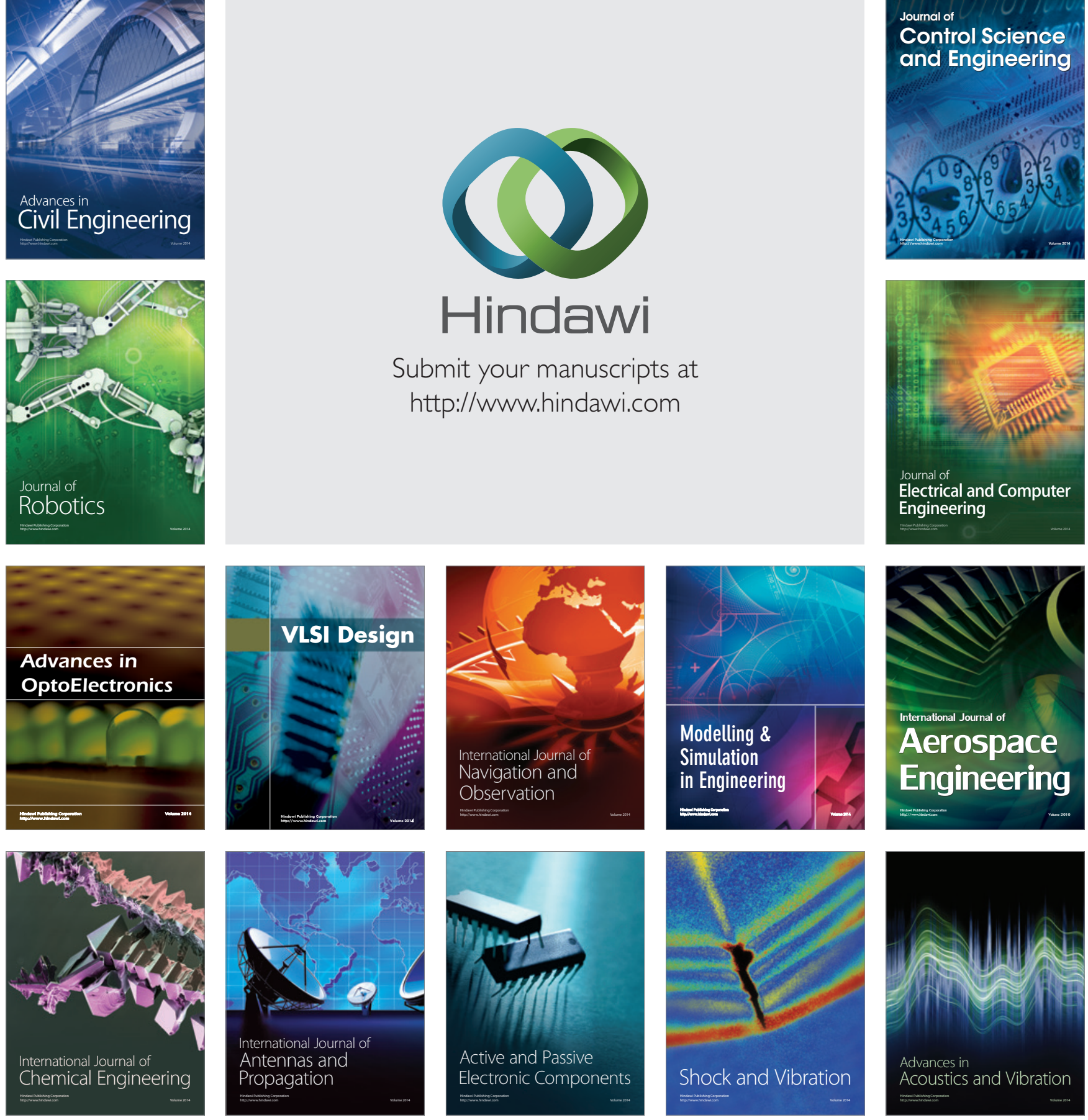